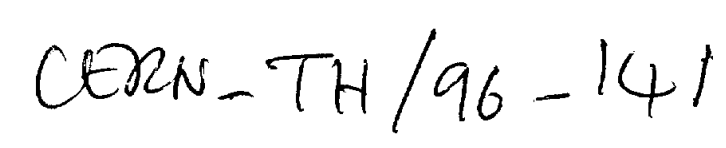

Contribution to the book honoring Andrzej Bialas

Fundamental Physics from Space and in Space

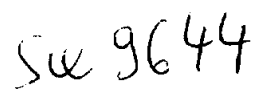

M. Jacob

CERN/DSU

CERN LIBRARIES, GENEVA

\title{
1. Foreword
}

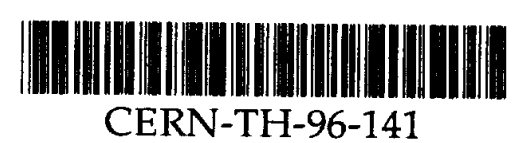

Andrzej Bialas is a long time colleague and friend. We have long shared the same interests in physics and it has always been greatly rewarding to interact with him, as we have done many times. It is therefore a great pleasure to contribute to this book which is honouring him. This I do not only for him but also for my long friendship with his country, Poland, which I have had the pleasure of visiting many times. Particle physics in Poland has long been closely associated with CERN and the Theory Division, to which Andrzej paid many visits, and which was long chaired by Jacques Prentki, benefited much from its welcoming many Polish colleagues and this over 4 decades.

My first contact with Poland goes back to one of my great uncles, Franck Schoell, who first translated "The peasants" of Reymont into French. Among his descendants may be counted many cousins of Polish origin due to several Franco-Polish marriages. I twice visited Andrzej's beautiful home town, Krakow, and the second time was particularly moving since it is there that my daughter had then just acted the Polish part of the "Double life of Veronica" by Krzysztof Kieslowski. Having him twice as a director has been, in her own words, a wonderful gift.

Over the past few years I have had to move away somewhat from full time theoretical physics research as other commitments, as they frequently come with age, have become very demanding. Yet I started at the same time to take great interest in fundamental physics in space in connection with new responsibilities with the European Space Agency. It can therefore be deemed appropriate that I cover this question here. Cosmic ray research and particle physics research have both a great tradition in Poland. Physics from space and in space should also eventually blossom in that country.

\section{Particle physics from space and in space}

At present, one notices an increasing interest in particle physics related to space. Indeed, the study of particles from cosmic sources appears to provide most interesting clues for questions originating from particle physics proper as well as from astrophysics. A new field of investigations has been born and is referred to as Astroparticle physics. Many particle physicists are turning to it. Some laboratories in particle physics are even concentrating a good fraction of their activities on this new domain. This may look peculiar since cosmic rays, which had long provided the only sources of very high 
energy particles, had been almost abandoned in the fifties for accelerators which could provide intense sources of high energy particles produced under specific conditions. These accelerators, and now colliders, have allowed one to collect impressive statistics on collisions of ever increasing energy. At CERN, the LHC will soon open the multi-TeV domain (14 TeV in proton-proton collisions) and should provide instrumental clues in our answering many fundamental questions. Poland, as a new CERN member state, is fully involved in this endeavour.

Progress in particle physics, through now already four decades of accelerator based research, has lead to a much better understanding of the structure of matter. This has revealed the quark structure and culminated, with the Standard Model, in a detailed and accurate description of all observed phenomena. This accelerator-based research has also provided a much better understanding of the Cosmos at large, and in particular of what happened just after the Big Bang. This has in turn opened up to investigation new and fascinating questions. As we understand the universe better, we realize that the shining mass, which is associated with radiant stars, is probably only a few per cent of the total mass in the Universe. The search for "dark matter" has thus become a great challenge. Clues are looked for with accelerator physics but also from space. Whereas the Big Bang was the most violent event ever, the present Universe, observed outside of the visible spectrum, also provides multiple examples of violent phenomena, producing very energetic particles.

Neutrino physics, now well understood through accelerator research, provides a unique way to study these phenomena through particles not affected by their long journey through space and also to look in the interior of the more quiet stars. Several underground (or underwater) detectors have been built, and more ambitious ones are being built, for the primary role of studying cosmic neutrinos.

In Europe, for instance, there is the important Italian-led Gran Sasso laboratory, already busy with many experiments which often correspond to large international collaborations. There is also the Greek-led Nestor project, an underwater array off the coast of Greece, now at the building stage and which has developed into an international collaboration. There is also the Swedish-led project Amanda, deep under the South polar icecap, and several others of lesser importance. There are also several other very large projects on the world scale, in North America, in Japan and in Russia.

The Standard Model still leaves Gravity aside but particle physicists are working hard to understand gravity better and to include it, with full legitimacy, in their global study of the basic interactions. We are still far from a quantum theory of gravity, even if superstrings may perhaps already provide insights on what the solutions in such a theory could be. As a first and most important step, collecting evidence for gravitational waves offers challenging prospects. The study of pulsar binaries leaves little doubt as to their existence as predicted in Einstein's General Relativity. The expected effects are however so tiny (in the $10^{-22}$ range) that detection is extremely 
difficult. Yet prospects, with new laser interferometers, are high. In Europe, for instance, there is the Franco-Italian VIRGO detector and a smaller project in Germany, both at the building stage. There is also the LIGO project in America. Some particle physicists are turning to this research.

Particle physics should be pride of this trend. It enlarges its scope and in a direction where public support seems at present easier to find than for the deep study of matter. The cosmos is full of (often erratic) accelerators. The detection techniques and the international collaborations required for experimentation in astroparticle physics bear much similarity with those on earth-bound accelerators. The funding agencies seem to welcome that. For instance, in France and in Italy, the CNRS-IN2P3 and the INFN respectively, which fund primarily particle and nuclear physics, have already much extend their activities toward astroparticle physics and jointly fund VIRGO, which represents a major investment. In Britain, Particle Physics and Astronomy, have been brought together within a unique funding structure, PPARC, which could be a natural niche for astroparticle physics if the funding envelope were not already very tight.

One can easily foresee an important extension of astroparticle physics over the coming decade. It will correspond to earth-based research even if it is turned to space. Looking a further decade or two ahead, one can however anticipate that part of this research will be turning from earth-based detectors to space-based ones. The cost of experiments in space is of course several orders of magnitude higher than on the ground, or even underground (underwater), at equal weight. However, going into space may become a must in view of the foreseen scientific return. At present, one may mention the AMS experiment, searching for antimatter in space using a permanent magnet spectrometer. It will be flown on the shuttle in 1998 before being installed on the space station in 2001.

One should also say that the results of COBE on the microwave background have triggered much interest among theorists for which the early universe provides the only experiment at their disposal to test some of their most fundamental theories. There is a great demand for a much higher resolution than the one available with COBE. The ESA project, COBRAS/SAMBA, which should fly in 2004 should provide it. For gravitational waves going into space is a must if one wishes to detect them in their highly promising low-frequency band. After its 1994 review, ESA has listed such a detector among its Corner Stone projects for its present longterm programme extending into the second decade of the years 2000 . The code name is LISA (Laser Interferometer Space Antenna). This will be discussed at some length later.

Considering fundamental physics in space, one can say that the cost of each experiment will imply a high specificity. At the same time, such ventures will often cut across traditional disciplines in physics and trigger the development of numerous enabling technologies. We already see there a budding but thriving new community of physicists at work. 
In this review I shall start with an overview of fundamental physics from space, as it is today, and later turn to fundamental physics in space, as we may already foresee part of it at present.

\section{Fundamental physics from space}

During the seventies, there was an important development of underground research. It was initially motivated to a large extent by the interest in Grand Unified Theories with the testable prediction of proton decay. The lower limit for the proton decay rate has been pushed above 10-32/ years (looking for the positron- $\pi$ zero mode) and, if proton decay was not observed, this type of detector turned out to be most rewarding for the study of cosmic neutrinos. New, larger and more sophisticated detectors have been built and are being built. Indeed, a major and very rewarding event was the observation of neutrinos from the collapse of the superNova 87-A in the great Magellanic cloud, 150.000 light years away. Few neutrinos were caught but in a number which tallies with expectations. Grand Unified theories also pushed for monopole search in cosmic rays but with no success, something which no longer comes as a surprise with inflation theories.

The question of dark matter has been approached from several sides. It is now clear that a sizable fraction of it (15\% of the critical mass) could be in the form of dark objects in the Galaxies, the so called MACHOS. They are searched for astronomically through gravitational lensing effects. This work, which implies very sophisticated data handling, is carried out in part by particle physicists. Such objects have been found.

A possible culprit for the missing mass to the full critical value, favoured in inflation scenarios, could be a small neutrino mass. Indeed a mass value of $30 \mathrm{eV}$ would be enough to close the Universe. The tau neutrino could be the one massive enough. This question is linked to that of neutrino oscillations and therefore to the electron neutrino flux received from the sun. Beautiful results were obtained from the Gallex experiment, in Gran Sasso, and from the Sage experiment, in Baksan. They may show a deficit between the number of neutrinos detected and those expected to be detected according to solar models, as if oscillations were depleting the electron-neutrino flux received on earth. However the discrepancy is expressed in SNU, a unit which folds the incident flux with the detection probability, the latter being a fast-rising function of energy. As a result minority processes which produce higher energy neutrinos, and about which one could have still some uncertainty, are overemphasized with respect to their contribution to the global energy output of the sun. The reported discrepancy is actually still small in terms of the global number of SNUs (one sigma) but it is stronger if one brings all experiments (with their different thresholds) into the picture, in order to try to pin down some particular contributions. One has then to rely on all present experiments which different people may qualify in different ways. If one really wishes to fit everything, it is possible but with neutrino oscillations corresponding to a mass square difference of the order of $10^{-5} \mathrm{eV}^{2}$. The present large neutrino oscillation experiments, such as NOMAD and 
CHORUS at CERN search for tau-mu neutrino oscillations. The mass range suggested by solar neutrino experiments for electron-mu oscillations would, be well-suited to the study of oscillations with a multi- $\mathrm{GeV}$ beam from an accelerator shot at an underground detector a thousand $\mathrm{Km}$ away, such as those which can be considered between CERN and Gran Sasso .

The question of neutrino oscillations is likely to remain a very topical matter for several years to come, combining accelerator particle physics and particle physics from space. From space we have the neutrinos from the sun, already mentioned, but also atmospheric neutrinos which also seem to cause problems.

With large underground or underwater detectors, and also under- ice detectors, one faces the question of very high energy neutrinos from space $(>1000 \mathrm{TeV})$. They provide unique information on very violent phenomena in the cosmos, which, in some cases, could be correlated with the observation of very high energy gamma rays. There are also the low-energy neutrinos associated with supernovae but the expected rate of those detectable is small. This is not a reason not to keep a watch with improved detectors. This is done.

The questions of very high energy photons, which could be associated with steady or erratic point sources in the universe, is also very interesting. Above $100 \mathrm{GeV}$ the atmosphere is a very suitable detector which comes for free. Significantly below that, one has to go to space. A study of photons from the Cosmos in the $10-100 \mathrm{GeV}$ range could well be among the first astroparticle physics experiment on the moon. There may be a jackpot there. Photons with well-defined energy may also come from the pair annihilation of hitherto unknown particles which would be stable remnants of the Big Bang. Supersymmetric partners of the known particles are among the candidates.

Astroparticle physics on the ground, or underground, is at present going through an important development. The first findings, but also some of the negative results, the dogs that did not bark, are a strong encouragement to continue.

At present the sophistication, size and cost of all these important underground (underwater) detectors is enough to keep all physicists involved in this research busy. The same applies to those involved with the development of large arrays on the ground. They cannot afford to be greatly involved in space based research and the same applies to their precious and hard-to-get funding which they would not like to see diverted to space research. Within a decade or two, the situation may change. There are already some ventures.

As already mentioned, one important exempla of present particle physics research in space is the AMS experiment. Its aim is to study antimatter in space with a sensitivity 4 to 5 orders of magnitude beyond what is presently known. The experiment will certainly detect antiprotons and 
positrons (expected from high-energy collisions in space) and gamma rays. It will also measure abundance ratios for light elements which are very important for nuclear synthesis studies. No signals for large amount of antimatter annihilating against matter has so far been detected up to the "local" super cluster level. Our present views about the Big Bang lead us to think that all antimatter was actually annihilated during the first second. Yet, it is worth checking, searching for antimatter with increased precision.

Ground studies of gravitational waves are also going through an important new development. In the framework of Einstein's General Relativity gravitational waves should exist and the evolution of pulsar binaries, such as the famous Hulse-Taylor one, fits very well with expectations. This can be considered as a proof, however still indirect, of gravitational wave radiation. The rates expected from specific sources, which can now be much better estimated, are however very low. The typical values of the (dimensionless) amplitude are at the level of 10-22, very much lower than the detection limit of the present oscillating bar detectors which have been constructed following the pioneering work of J. Weber $\left(10^{-18}\right)$ at present. However, following this time the pioneering work of $\mathrm{R}$. Weiss, $\mathrm{Km}$ arm length laser interferometers should reach a detectablility level corresponding to known sources such as supernova collapse and compact binaries coalescence. This is what is attempted with the VIRGO project in Europe and the LIGO project in America. Prospects are high to detect gravitational waves with these detectors which will be used in coincidence ( 2 in America and 1 in Europe). Ground-based detectors are most efficient in the 10 to $10^{3} \mathrm{~Hz}$ range but they are blind at low frequencies $(<10 \mathrm{~Hz})$ because of gravitational noise on the earth. This higher frequency domain is where we expect signals from supernova collapse and from the end stage of compact binaries as they eventually coalesce (the last 15 minutes before merging and ringdown). This is very interesting but one has to be lucky because of the not-so-probable occurrence of such events even if one reaches a detection level corresponding to our near by cluster of galaxies, Virgo. The signal from a supernova is hard to estimate; but templates will help with coalescence search. In all cases such signals come only once and are very brief. One has to rely on coincidences between different detectors to be sure. Stable signals which are expected from stable binaries are in the low-frequency range, something which requires detection in space. We shall come back to that. In any case we see at present the development of a thriving community focusing on the detection of gravitational waves. The first signals, which one can now strongly hope for over the coming decade, and which could be detected on the ground, will be a strong encouragement to move to space.

\section{Fundamental physics in space}

One may try to anticipate what fundamental physics in space will look like up to two decades ahead using, as a guide, the ESA's future long-range study. The sophistication and cost of the necessary instruments are indeed such that those which may be available by then have already to be discussed 
today. The ideas and aspirations are those of the scientific community. In 1993, ESA made a call for mission concepts in order to prepare its Horizon $2000+$ programme. This was intended to be a rolling-forward continuation of its Horizon 2000 programme as it had reached mid-term. The combination of the two programmes should define the key elements of space research in Europe up to the second decade of the next century. At this stage one is often not discussing actual fully-fledged proposals, which will probably use a technology still to be developed, but mainly general ideas about what should be attempted and how. There are refereed to as mission concepts.

The call for mission concepts resulted in 110 proposals presented to the Survey Committee. They were classified according to 5 general categories and Topical Teams were set up to assess them. These topical teams were:

1 Moon, Planets and small bodies.

2 Sun, heliosphere and plasma physics.

$3 \quad$ High-energy astrophysics.

4 UV, Optical, Infrared, Radio astronomy.

$5 \quad$ Fundamental physics.

This was the first time that "fundamental physics" appeared separately within the ESA planning. There was a need for that. About 30 of the 110 proposals were falling under that heading! Fundamental physics has many facets. Those considered more particularly fell under the general headings of cosmology, gravitation and particle physics. The question of microgravity experiments was reviewed separately, as something which should rather be linked with the space station, considered as a separate entity.

ESA classifies its missions as "cornerstone"-type, medium and small. A Cornerstone mission is a major one, at the level of up to a Billion CHF at the present rate. It is also a mission which ESA could carry out all by itself. There is of course the possibility and hope that other agencies could contribute to make it better and quicker but, even if this should fail, ESA would commit itself to do it fully. We see here an interesting parallel with the LHC which the CERN Member States have agreed to build by themselves but hoping that significant contributions by non-Member States will allow them to construct it better and faster.

A medium-size mission corresponds to about half the cornerstone value or could be a larger mission in which ESA is only a partner. In Europe smallsize missions are usually considered in the framework of national agencies.

There are 4 cornerstones in the Horizon 2000 programme. There is the Soho/Cluster complex, with Soho already in orbit and Cluster soon flying with the maiden flight of Ariane-5. This falls in the area of topical team 2. There is Rosetta, a mission to comet Wirtanen, which will fly in 2003. It will follow the comet and send two landers on it. This belongs to the area covered 
by topical team 1 . One also has XMM, an X-ray observatory, which will fly in 2000. It belongs to the area of topical team 3. There is finally FIRST, a mm wavelength observatory to study the cold universe. It should fly in 2005 and falls in the area of team 4. Among the cornerstones added for the programme Horizon 2000t, there is, for the first time, one in fundamental physics. This is LISA, a space interferometer for the study of gravitational waves. It should however fly last, yet, if everything goes well, before the end of the second decade of the next century. This is a time of solar minimum if one wishes to find comfort for such a late date. The other two are an orbiter mission to Mercury and an interferometric observatory which will provide a 10 microarcsecond resolution, respectively.

Fundamental physics has thus made its entry in the long-term space programme of ESA. Over a shorter timescale, one may also now have strong hopes for an ESA participation in a much smaller mission which could test the equivalence principle at the level of $10^{-17}$ to $10^{-18}$.

The relation between astronomy and fundamental physics is interesting. After the success of COBE, the COBRAS/SAMBA mission was classified as an astronomy mission and it eventually met success that way. It may well be that before COBE, defending such a mission would have been the privilege and task of a fundamental physics advisory group, something which the present one did anyway. It may be that, 30 years from now, the successor of LISA will fall under astronomy, as providing a very precious and unique tool for the observation of very massive black holes. But, today, with all the uncertainties attached to such an endeavour, the detection of gravitational waves falls under fundamental physics. This is actually proper. We are concerned not so much about the detection of gravitational waves which should be certainly detected if they exist, but about understanding their production and properties and in particular in the high-field regime.

Coming back to the ESA review of 1994, the team confronted with the proposals falling under the heading of fundamental physics had much to study and assess. I was much honoured to chair that group as a neutral nonspecialist scientist but I had the help of very fine specialists who were the other members of the group: I. Ciufolini, T. Damour, G. Schäfer and B. Schutz. There were also J.P. Blaser, assuring the contact with the Survey Committee, and G. Tammann, for the contact with Astronomy. The most useful and knowledgeable secretary of the team was R. Reinhard from ESA.

What are the goals of fundamental physics in space now? The best way is to define that is to do it according to the proposal received. We could itemize 6 different entries. They are the following:

(i) Tests of the equivalence principle. There were 3 proposals.

(ii) Tests of the Newton law. There were 2 proposals.

(iii) Particle physics. There was 1 proposal (on cosmic background neutrinos) 
(iv) Gravitational waves. There were 2 proposals, easily merging.

(v) Search for long-range spin dependent interactions. There were 3 proposals.

(vi) Qualitative tests of the Einstein Theory. There were 10 proposals.

There were still other proposals but of a more technological nature. They were often aimed at testing new technologies needed for the realization of some of the scientific missions falling under these 6 entries. Identifying socalled "enabling technologies" has indeed much to do with any recommendation.

The outcome of the work resulted in 5 main points, namely:

(i) Recommending one cornerstone mission associated with the detection of gravitational waves. This is LISA.

(ii) Discussing the relative merits of the space and ground base detection of gravitational waves.

(iii) Highlighting 3 research directions which appeared as particularly promising among those present in the proposals. They are: the detection of gravitational waves, the test of the equivalence principle at a level 5 orders of magnitude beyond the present limit of $10^{-12}$, and the test of the relation between mass and curvature and in particular the spin content of the gravitation interaction, respectively.

(iv) Evaluating separately most of the proposals.

(v) Assessing enabling technologies for the missions which were relevant to point (iii).

At the time of the survey, a mission focusing on the test of the equivalence principle, but also searching for long-range spin dependent forces (an axion flavour) and involving a precise geodesy programme, was under study as a candidate for the M3 (M for medium) mission, M3-STEP. There was therefore not much more to say along that line. It was not kept eventually in the selection which retained COBRAS/SAMBA. There is however a strong hope that a less ambitious mission, which would limit itself to a test of the equivalence principle at the $10^{-17}$ to $10^{-18}$ level, will be eventually flown at a much lesser cost, benefiting from equipment already developed in another agency. I will be achieved in a cooperative framework.

About the relation between mass and curvature, one may say that Einstein Theory introduces 3 post-Newtonian parameters. One of them, is alpha, which links the proper flaw of time to the gravitation potential. An other one, gamma, measures the spin content of the gravitation interaction. Its value is 1 in Einstein Theory which corresponds to a pure spin 2 exchange 
when considered as a field theory. The possible difference between gamma and 1 could be tested to the precision of $10^{-7}$, when the present limit is $10^{-3}$. This is important since some theories call for a scalar mixture which could have dropped down with the expansion of the universe.

The test of parameter gamma is still at the level of ideas. We thus now concentrate on the detection of gravitational waves. ESA has now included it in among its Cornerstones. If everything goes well, this programme should be achieved by the end of the second decade of the next century (Launch in 2017, say).

\section{The detection of gravitational waves}

The principle of the measurement is the same as the one of VIRGO/LIGO. It is based on a Michelson interferometer using laser beams. Going to space, one can focus on the detection of long wave length radiations (frequencies of the order of $10^{-3}$ to $10^{-2} \mathrm{~Hz}$, say). The optimal arm length is then of the order of 1 million $\mathrm{Km}$ but, in space, space is for free. Transponders, in phase with the incident laser beam, will replace the mirrors used on the ground where, despite Perrot-Fabry interferometry, available distances imply focusing on the shorter wave lengths (frequencies of the order of $10^{+2}$ to $10^{+3} \mathrm{~Hz}$, say). A ground experiment suffers anyway from inescapable gravitational ground noise level at low frequencies (below $10 \mathrm{~Hz}$ ). It is "blind" to low-frequency signals. In spaces, on the contrary, no such noise is present. There is some drag noise which can be compensated and this becomes worrisome only down to the level of $10^{-4} \mathrm{~Hz}$. It is therefore natural to optimize a space detector to the lower frequency domain, leaving the higher frequency one to the less expensive ground detectors. One therefore sees that there is a good complementarity between ground-based and space-based detection. One can thus cover the $10^{-4}$ to $10^{-1} \mathrm{~Hz}$ band in space and the 10 to $10^{+4}$ one on the ground.

It is clear that when faced with a new phenomenon it is important to study it over as large a band width as possible. Indeed, the long praised quietness of the heavens is misleading and it reflects observations long limited to the optical visible spectrum. There is more to it with gravitational waves.

The high-frequency ones (typically $10^{+3}$ in that case) find their natural sources in sporadic events such as the asymmetrical collapse of a supernova or the final coalescence of a compact binary. Such sources exist but, if one wishes to have a decent probability of observation at the level of one year, the detection sensitivity should match sources all the way to the nearby cluster of galaxies, Virgo, which is about 30 Million light years away. These sources emit strongly but during a short time; A brief instant for a supernova and 15 minutes for a coalescence. One has to have the detector "on" at the right moment. Coincidences between different detectors is then necessary to eliminate spurious noise. This being set up, the joint response provides direction and polarization information. 
The low-frequency waves (typically $10^{-3}$ in that case) are emitted by compact binaries (black holes, neutron stars, white dwarfs....) in their stable regime. Their radiation extends steadily over eons. The Hulse-Taylor binary will coalesce only $10^{+8}$ years from now! The low-frequency waves also correspond to the formative stage of very massive black holes or to the coalescence of two very massive black holes (several million times the solar mass, say) which also cover a rather long time. The typical emission frequency of such objects is indeed inversely proportional to their mass, with $10^{-3} \mathrm{~Hz}$ for 10 times the solar mass providing the normalization. In the former case, there are plenty of sources in the galaxy and space detectors could most likely see tens of thousands of them. In the latter case, the signals are so strong that they could be detected up to a very large distance, well beyond the "local" super cluster level (200 million light years). This should provide an observation probability even if such events are very rare at the level of a few galaxies. Having thus access to the very massive black holes would open a new field of investigation for astronomy. Such objects are expected to exist at the center of most of the galaxies and galaxy collisions are not very infrequent. As previously hinted at, the second space gravitational wave detector may thus well fall under the heading of "astronomy" and no longer under "fundamental physics". As previously said, this however should not belittle the fundamental physics interest which will very much remain. Studying gravitational wave emission in the strong field relativistic regime of very massive black holes should provide many clues for a better understanding of gravitation. Emission in the low-frequency domain should extend over a reasonable to extremely long length of time. There is therefore no need for two detectors operating in coincidence. As the detector circles the sun it will provide, through Doppler shift, information on the direction of the source. It will also determine the polarization, through its slow tumbling.

The observation of gravitational waves would be a dramatic and fundamental discovery. For many reasons, it will probably take place on the ground, during the first decade of the next century. However, if nothing is found, one could put the blame on lack of luck or on crude astrophysical models used at estimating the signals. In both cases, it will make it urgent to go into space, either to explore the rich low-frequency range or, in the latter case, to also first really test Einstein 's Theory. Indeed strong enough stable and predictable sources at low frequency do exist. There are certainly many binary systems in our galaxy. Failure to observe them would be a serious problem for our present gravity theory.

It is therefore proper to prepare to go into space before ground-based results are available. There is actually a good overlap between the groundbased and space-looking communities; and experience with laser interferometry on the ground will be beneficial for the space detector. The space detector should be continuously corrected for drag, correcting erratic motions of the shell as compared to the continuous free fall of the active payload. This calls for important technological developments which should be started early. Sensors and thrusters have to be designed and built. 
Gravitational background noise associated with the early universe or with cosmic strings should extend over the whole covered spectrum ( $10^{-4}$ to $10^{-1}$ in space and 10 to $10^{+4}$ on the ground). The size of the signal is however still speculative. One expects the frequency range of observable gravitational waves to extend from $10^{+4}$ (formation of a black hole of a little over a solar mass, just at the Chandrashekar limit) down to $10^{-18}$ (when associated with quadrupole deformations at the level of the whole microwave background).

LISA would consist of 6 spacecrafts, 3 sets of 2 . Within one set the lasers on board will be phase-locked and act as a single laser with beams in phase sent in two different directions making an angle of 60 degrees. One could make do with a single craft but having two independent spacecrafts makes orientation much easier to justify the only apparent extra cost. The two space crafts would be $200 \mathrm{Km}$ apart. Each set of spacecrafts is at the apex of an equilateral triangle with a side length of 5 Million $\mathrm{Km}$ (figure 1).The laser beams from each set are directed to two of the distant spacecrafts and interference between the beams received from the transponders is used at detecting minute phase changes between the beams received from the two arms. The rest of the system (with the 6 spacecrafts in total) is here as an active back-up, providing redundancy in case one element should fail, but also giving information on polarization. The detector would trail the earth at 20 degrees on a solar orbit (figure 2). It should be far enough not to be appreciably perturbed by the earth-moon motion. It cannot be too far because of cost containment. The plane of the detector is chosen so as to minimize the slow changes of lengths as the whole system circles the sun and tumbles. The relative pulsative variations in lengths which could be detected are at the level of $10^{-23}\left(10^{-3}\right.$ to $\left.10^{-2} \mathrm{~Hz}\right)$. This is also what is eventually aimed at with ground-based detectors (VIRGO, LIGO) but, now, in the $10^{+2}$ to $10^{+3}$ frequency range. The limit in the optimal frequency ranges comes in both cases from laser photon shot noise. On the ground this also cuts off the sensitivity level on the high-frequency side, whereas seismic noise and thermal noise cuts it off abruptly at low frequencies. In space, the drag noise provides the sensitivity limit at low frequency $\left(10^{-4} \mathrm{~Hz}\right)$, whereas the arm length provides the dominant natural sensitivity limit at higher frequency $\left(10^{-1} \mathrm{~Hz}\right)$.

\section{Some of the expected signals}

We have good reasons to believe that a sensitivity at the level of $10^{-22}$ to $10^{-23}$ should be enough for many interesting sources. One may remark that it is much higher than that achieved with resonating bars (at frequencies in the $10^{+3}$ range), for which the present limit is $10^{-18}$. Whereas some sources could be detectable with bars, some luck would also be needed. With laser interferometry one has a very significant advance which may be lengthy and costly to reach with cryogenic bars. Detecting a relative change at the level of $10^{-22}$ over 1 Million $\mathrm{Km}$ amounts to measuring a displacement of a thousandth of the size of an atom within the spacecraft. Laser interferometry should do that. It presently already does much better on the ground. To put it 
in a nutshell, one "sits" on a black fringe and collects the few photons which might nevertheless escape as the phase difference between the two beams changes by minute amounts.

Gravity is a non-linear theory. Gravitational waves are ripples in the curvature of space time which propagate at the speed of light but separating the curvature ripples from the rest represents an approximation. Let us assume that we can do it. One can then define a two-dimensional tensor hij the second derivative of which with respect to proper time will be proportional to the iojo component of the contribution to the curvature tensor associated with the passing wave. The space directions ij are transverse to the axis of propagation. The basic gravitational radiation is of a quadruple type and the amplitude is therefore describe by a tensor hij. The gravitational force is directly linked to the curvature tensor contracted with the probing length. So is the acceleration, hence the second time derivative in the relation of $h$ to the curvature tensor. Integrating twice over time while assuming that the displacement is very small as compared to the arm length, which is always the case, one easily checks that $h$ relates the relative length variation along $i$ measured between two points separated along the $\mathrm{j}$ direction, both perpendicular to the direction of propagation. The test distance is here assumed to be small as compared to the wave length and to any overall effect of the curvature. The amplitude of the gravitational wave is given by $h$, which, as defined, is a dimensionless quantity. It decreases from the source being inversely proportional to the distance. It is a tensor associated with the quadruple nature of the wave but for simplicity we just considers an amplitude. The displacement effect is proportional to the test length.

Sources and expected effects are analysed in detail in the contribution of $\mathrm{K}$. Thorne to the book "300 years of gravitation". We merely quote some of the results here. When considering observation limits for $h$, one has to distinguish between burst and stable sources and also the frequency range and the time of observation. We shall not go into that here and merely quote the relevant values including such effects according to the different cases considered.

We first consider a supernova. Visible ones appear in the galaxy with a frequency of one every forty years. They could be more but, if we wish to have a reasonable chance to see anything during the first year of operation, we have to reach to the Virgo cluster (a thousand galaxies) and estimate the effect of a supernova collapse there, with $h$ a hundred to a thousand time less than it would be for a galactic supernova. Its value (inversely proportional to distance) is proportional to the square root of the non-symmetrical kinetic energy associated with the collapse, which is only capable of creating a quadruple radiation. It is also inversely proportional to the square root of the frequency. For a frequency of $10^{+3}$, at Virgo and with the solar mass as a unit, the overall proportionality ratio is of the order of $10^{-20}$. If the non-spherical collapse involves only $10^{-4}$ solar mass, the amplitude is of the order of $10^{-22}$. One therefore sees that the detector should aim at least at that. This is what is expected of VIRGO/LIGO, in their second generation development. The sensitivity should be pushed down to $10^{-23}$ in that frequency range. 
Let us now consider formatting or better coalescing black hole(s). The typical frequency is, as previously said, inversely proportional to the mass. The value of $h$ is proportional to the mass. The formation of a very massive black hole $\left(10^{+6}\right.$ solar mass) will therefore be much more visible than that of one of a few solar masses. It is less frequent at the level of Virgo but one can extend detection much further. The value of $h$ is proportional to the square root of the relative amount of mass involved in the event. If one takes this to be $10^{-2}$ and place the event in Virgo and consider a black hole of 10 solar masses, the overall coefficient is $10^{-20}$. With the sensitivity achieved such a black hole event could be detected on the ground $\left(10^{+3} \mathrm{~Hz}\right)$ if 0.01 for the relative amount of mass creating the radiation is the right order of magnitude. Very massive black holes ( $10^{+6}$ solar masses) should, however, be easily seen in space and from anywhere in the visible universe.

For binaries the value of $h$ at the coalescing time should be of the order of $10^{-21}$ in Virgo. This should be observable on the ground at high frequencies $\left(10^{+3}\right)$. The radiation level during their long stable life is much smaller and one has to go to the Galaxy to get as high a value for $h$. If the galaxy may not be enough to catch a coalescence within one year and one has to watch in Virgo, it provides sufficiently many stable sources visible at low frequencies $\left(10^{-3}\right)$. One sees again the great complementary between ground-based and space-based detection.

Figure 3 shows the expected values for $h$ for some sources together with the sensitivity of LISA. Many sources should be within reach. It also shows the values expected from other sources at high frequencies. They should be within the reach of VIRGO/LIGO.

\section{Conclusion}

Fundamental physics using cosmic sources offers many exciting prospects, whether it is considered on the ground or in space. In particular the observation and study of gravitational waves appears at long last as within reach with a most interesting complementary between ground-based and space-based detection. In particle physics we are now used to a long waiting time between dreams and reality; and physics in space extends that further. Nevertheless its interdisciplinary aspect is such that fascinating problems also appear on the way, developing the necessary detectors. They will certainly trigger interesting spin-off because of the complexity and difficulty of some of the required technological developments. We may then still meet enthusiasm for projects which will allow observation only 20 years from now. It is this enthusiasm which I try to pass on in this review.

Further readings (instead of references)

For astroparticle physics

L. Maiani "Underground facilities" in "Large Facilities in Physics", WSPC (1995) M. Jacob and H.Schopper, editors. 
For gravitational Waves.

K.Thorne in "300 years of gravitation". S. Hawking and W. Israel, editors, CUP,1989.

K.Thorne. "Gravitational waves" Proceedings of the Snow-Mass 95 Summer Study on Particle and Nuclear Astrophysics and Cosmology, WSPC

G. Fontaine "Ground based detection of gravitational waves, in Large Facilities in Physics" op.cit.

K. Danzmann "Laser Interferometry Space Antenna (LISA) for gravitational wave measurements" in "Large Facilities in Physics" op.cit.

Horizon 2000+, ESA-SP-1180

Figure Captions:

Figure 1. The Lisa set up with its three sets of two spacecrafts.

Distances within one set and between sets are at the level of 200 and 5MIL.Km, respectively.

Figure 2. The Lisa detector in its heliocentric orbit.

Figure 3. The sensibility of Lisa together with the signals expected from specific sources. 


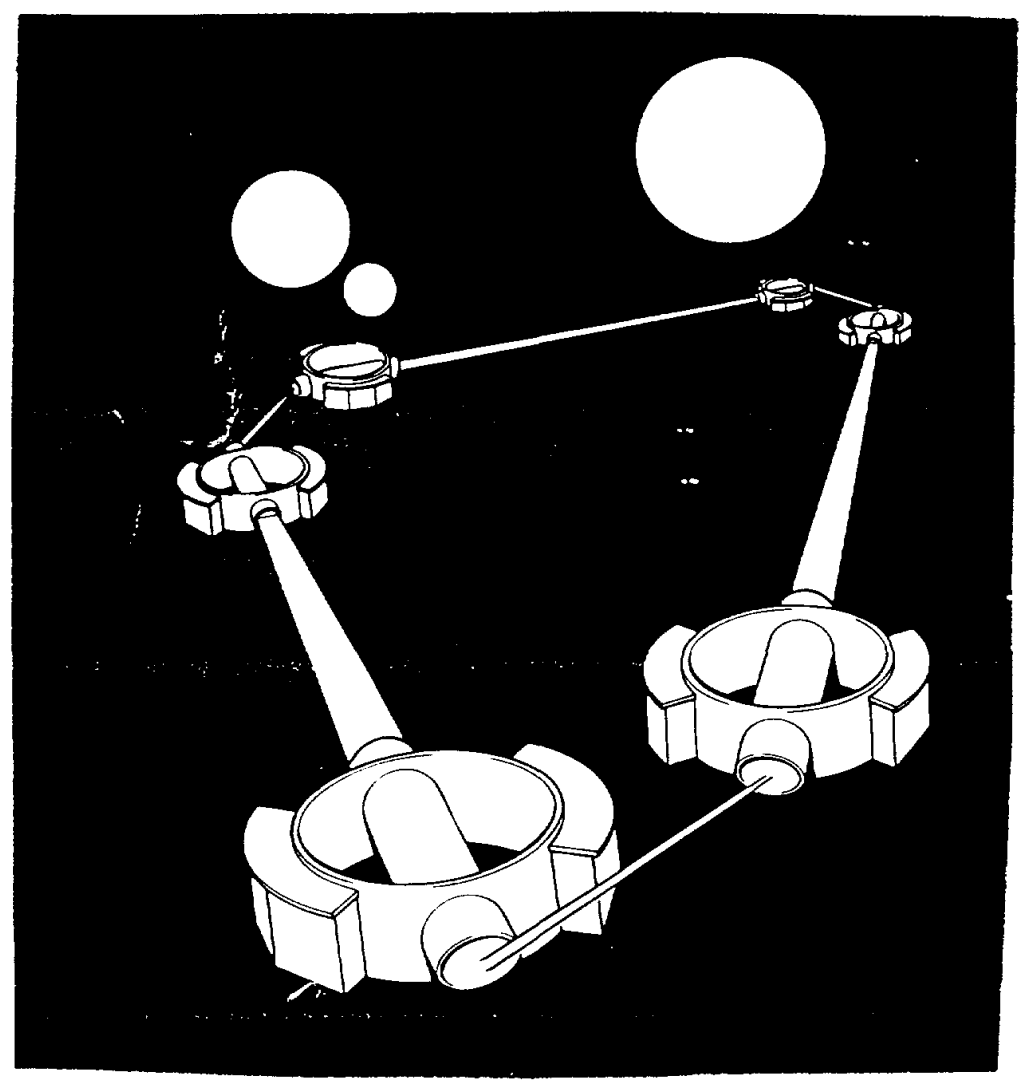

Figure 1. An artist view of the LISA set up with its three sets of two spacecrafts each. Distances between each set are of the order of 5 MIL Km. 


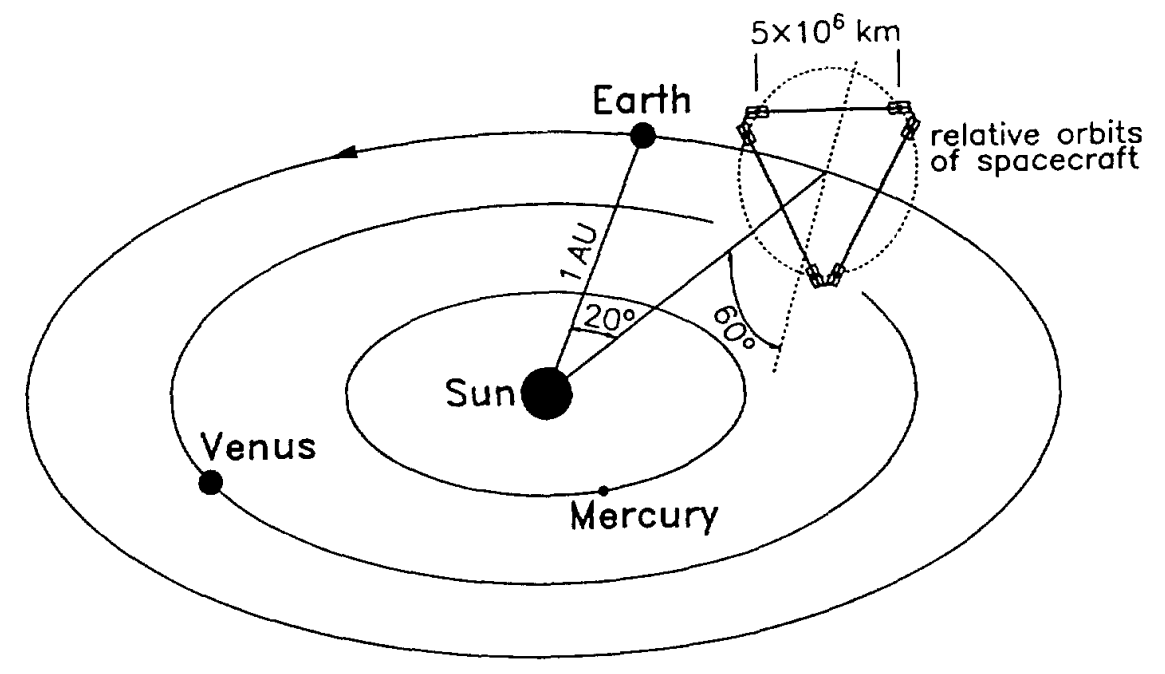

Figure 2a The LISA detector in its heliocentric orbit.

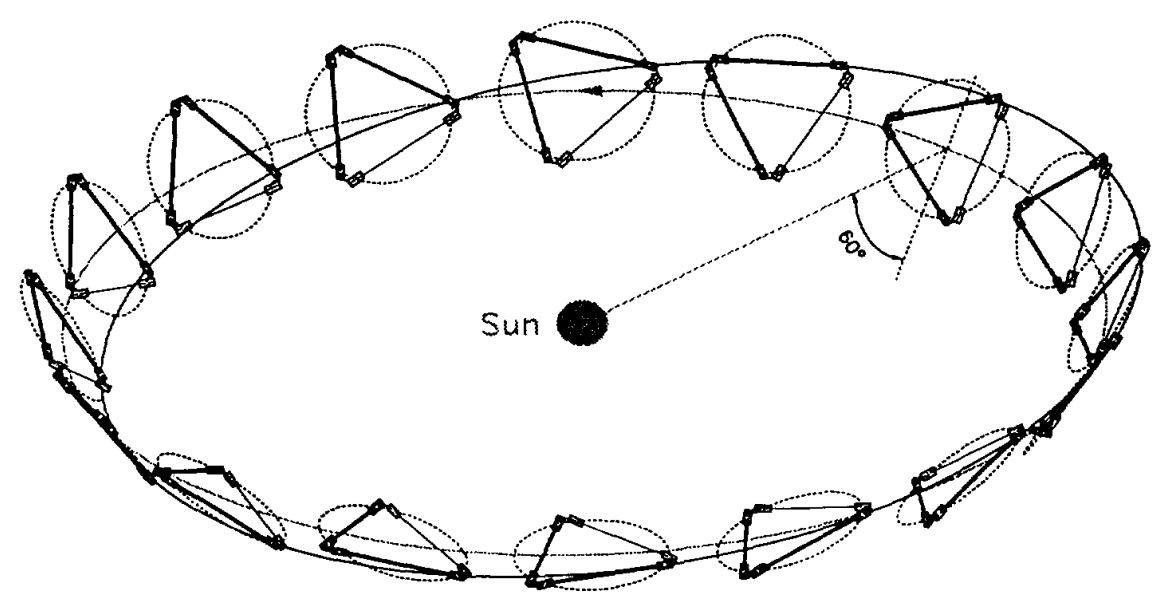

Figure 2b. The tumbling of the detector as it circles the sun. This allows one to determine direction and polarization. 


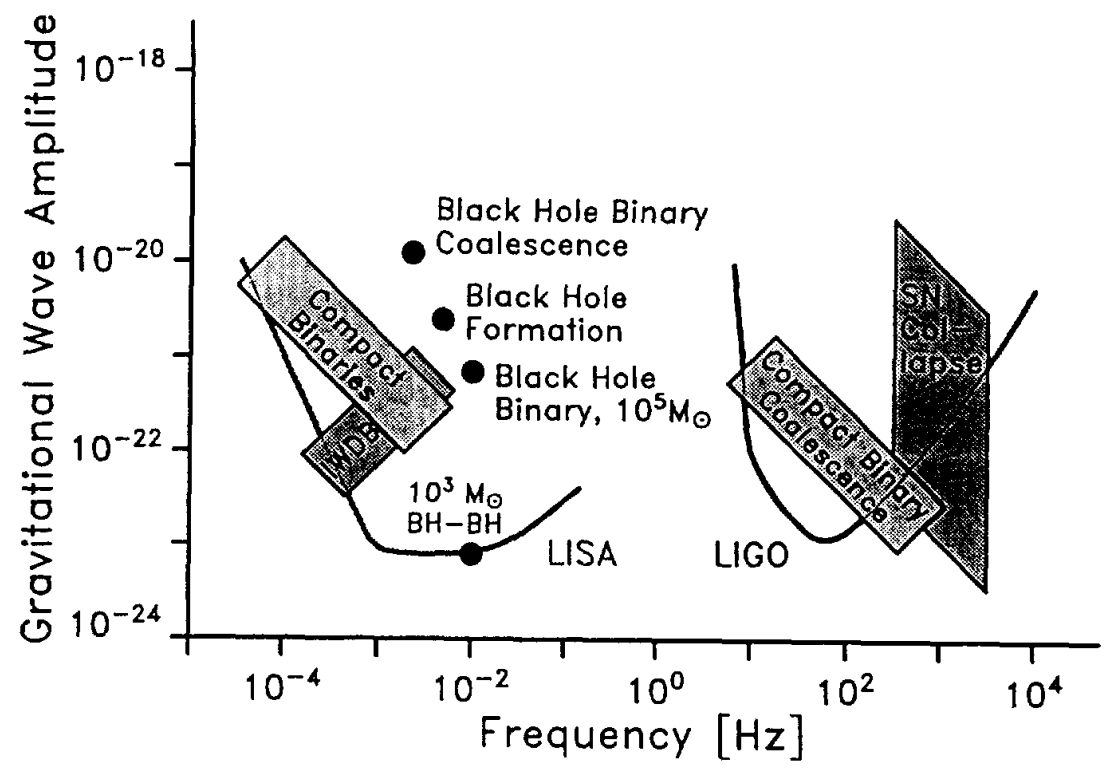

Figure 3. The sensitivity of the LISA detector together with expected signals from specific sources.

ALso shown is the same for ground based detectors (LIGO or VIRGO), now in the higher frequency range. 